\title{
La folie de l'arc-en-ciel ou la longue errance de Bwila
}

Les éléments organisateurs des remèdes de la folie des zu chez les Gouro de Côte d'Ivoire

Rainbow madness or the long wanderings of Bwila and the organisational elements of remedies for "zu madness" among the Guro of the Ivory Coast

\section{Claudie Haxaire}

\section{OpenEdition}

Journals

Édition électronique

URL : http://journals.openedition.org/ethnoecologie/797

DOI : 10.4000/ethnoecologie.797

ISSN : 2267-2419

Éditeur

Laboratoire Eco-anthropologie et Ethnobiologie

\section{Référence électronique}

Claudie Haxaire, "La folie de l'arc-en-ciel ou la longue errance de Bwila », Revue d'ethnoécologie [En ligne], 1 | 2012, mis en ligne le 02 décembre 2012, consulté le 19 avril 2019. URL : http:// journals.openedition.org/ethnoecologie/797 ; DOI : 10.4000/ethnoecologie.797

Ce document a été généré automatiquement le 19 avril 2019

\section{cc) (i) $(9)$}

Revue d'ethnoécologie est mis à disposition selon les termes de la licence Creative Commons Attribution - Pas d'Utilisation Commerciale - Pas de Modification 4.0 International. 


\section{La folie de l'arc-en-ciel ou la longue errance de Bwila}

Les éléments organisateurs des remèdes de la folie des zu chez les Gouro de Côte d'Ivoire

Rainbow madness or the long wanderings of Bwila and the organisational elements of remedies for "zu madness" among the Guro of the Ivory Coast

Claudie Haxaire

«Si nous nous représentons la vie de l'individu comme une pièce plus ou moins grande, il devient clair que presque tous n'apprennent à connaître qu'un coin de cette pièce, cette place devant la fenêtre, ce rayon dans lequel ils se meuvent et où ils trouvent une certaine sécurité. Combien plus humaine est cette insécurité, pleine de dangers, qui pousse les prisonniers dans les histoires de

Poe, à explorer de leurs doigts leurs cachots terrifiants, à tout connaître des frayeurs indicibles

qui en viennent!»

Rainer Maria RILKE (Lettres à un jeune poète)

\section{Introduction}

Si la majorité des ethnopharmacologues ne prennent que partiellement en compte le «binôme ethnique » (Kerharo \& Bouquet 1950; repris par Kerharo \& Adam (1974) de cette discipline, reconnu par leurs pères (Boiteau 1972) ${ }^{1}$ en France $^{2}$, ce dont témoignent les recensions menées par Etkin (2001), nous devons reconnaître, qu'en général, les ethnologues, de leur côté, explorent peu le «binôme naturaliste " ${ }^{3}$ À l'instar d'EvansPritchard (1972) en effet, nombre d'anthropologues travaillant sur l'infortune et la maladie ont renoncé à suivre leurs interlocuteurs dans le détail des propriétés concrètes ou symboliques des plantes utilisées par les guérisseurs. A fortiori, malgré les magistrales 
démonstrations de cette nécessité par Lévi-Strauss (1990), les anthropologues de la religion ou de l'art négligent-ils bien souvent de rechercher la signification des objets naturels utilisés en la replaçant dans le savoir encyclopédique de la société étudiée. Pour ce qui concerne les rituels, Turner (1972) posait pourtant lui aussi comme nécessaire, pour l'interprétation du sens latent des symboles rituels, d'analyser ce sens en relation avec les autres contextes rituels et pragmatiques où ils apparaissaient. Pour ces derniers contextes, les soins de santé donnent l'occasion de constituer un corpus de données privilégié, car dans les sociétés non dualistes, nous ne trouvons pas de solution de continuité entre traitement « empirique » et mise en place de rituel, les uns prolongeant les autres, ce qui a été montré pour les soins des nouveau-nés (Haxaire 2007). Réclamée donc par différents courants successifs de l'ethnologie, cette intégration à l'analyse des connaissances encyclopédiques des peuples étudiés peine à se mettre en place, peut-être faute d'une vision de l'intérieur de ces connaissances, donc de leur organisation et des relations qui les lient.

Dans sa tentative d'appliquer une démarche herméneutique à l'anthropologie des pratiques (ici thérapeutiques), Loux, selon Saillant (1990), loin de se limiter au cumul des faits, « analyse les relations structurelles entre eux ». En matière de thérapeutique, Loux et Saillant (1995) cherchent à retrouver les principes organisateurs qui donnent accès aux logiques symboliques ${ }^{4}$, cela dans le but d'appréhender le "système médecine » du début du siècle en France pour la première, du Québec à la même époque pour la seconde.

3 Ayant la chance de pouvoir travailler sur un système dynamique se renouvelant en ma présence, j'adopterai le chemin inverse et ferai jouer à cette analyse le rôle de validation, confirmation ou critique, par les pratiques, des interprétations issues d'entretiens et d'observations d'épisodes de maladie et de rituels thérapeutiques présentés au préalable. Bien entendu, cette démarche, ici décomposée, s'est déroulée selon des allers et retours tout au long de l'enquête. Ces « principes organisateurs » ou cette logique symbolique ne sont jamais clairement explicités comme l'a noté Héritier (1996) pour ce qui est de la logique des humeurs, ils fonctionnent par prétérition. Allant de soi, il se peut néanmoins que l'on y fasse allusion au détour d'une recette, comme on s'en rendra compte. L'analyse de ces recettes médicinales est donc précieuse.

\section{Présentation du terrain}

4 Cependant, m'inspirant de F. Loux et F. Saillant pour cet article, je ne peux oublier les conseils méthodologiques que Françoise Loux donnait à ses étudiants, à savoir expliciter le lieu d'où ils parlent et les implications, pour eux, de cette recherche. J'écrirai ainsi à la première personne et m'interrogerai sur mon investissement tardif dans l'analyse des rituels thérapeutiques dans une recherche portant sur la maladie.

5 Tenir la gageure de travailler pendant des années sur la maladie en pays gouro tout en s'interdisant de développer une recherche spécifique sur la divination demande en effet interrogation. Cela d'autant plus que j'avais conclu une dernière enquête en pays gbaya sur la nécessité de replacer l'étiologie de la maladie dans l'ensemble de celles du malheur et de l'infortune. Certes Ariane Deluz, que j'avais autrefois rencontrée avant d'entamer une enquête sur le terrain qui lui était familier, s'inscrivait dans le champ de l'ethnopsychiatrie, se voulant héritière de Georges Devereux et ces rituels concernaient ce champ. Mais notre accord de «bon voisinage » d'alors portait sur une non intrusion 
dans ses enquêtes sur l'initiation féminine (Deluz 1987), ce que j'ai respecté. J'anticipais donc ses souhaits. Au reste, n'ayant pas compétence en psychanalyse, j'entends bien m'en tenir à la description ethnographique des entités rattachées par les Gouro aux différentes folies et maladies de la tête. Tout au long de ces années, au gré des parcours des malades avec lesquels je travaillais, je ne pouvais bien évidemment éviter de noter des histoires de consultations, des récits étiologiques contradictoires, d'identifier les devins, d'assister à leurs danses et d'enregistrer leurs chants, mais je ne m'attachais pas à explorer ce domaine plus avant.

6 Comme souvent dans mon itinéraire de chercheur en pays gouro, ce fut l'un de mes interlocuteurs, et non des moindres, Gonin-bi-Zre (de Danangoro), qui me mit en demeure d'investir cette question.

7 L'organisation sociale gouro est de type segmentaire, les unités territoriales étant autrefois en relation d'alliance ou de guerre. Je réside chez les Wagye et parcours plus volontiers avec eux la région nord où ils ont traditionnellement noué des alliances. Mais, depuis la colonisation et la scolarisation qu'elle a mise en place, des amitiés personnelles sont nées entre jeunes indépendamment des alliances anciennes. C'est ainsi que je fis connaissance d'un ami de lycée de Benin-bi-Dje, mon interprète, originaire d'une unité territoriale voisine, les Guo, mais issue de migration d'un groupe du sud, qui ne faisait pas initialement partie de l'aire matrimoniale wagye. C'est dire que mes compagnons n'avaient qu'une connaissance partielle des membres de cette communauté.

Cet ami, Gbo, était le fils de Gonin-bi-Zre. Il accourut, lorsque, pour venir à bout d'une nouvelle crise de «folie » (gwée) d'un jeune homme du village voisin, l'on fit appel à son père, grand spécialiste des maladies de la tête, que l'on donnait pour puissant féticheur. Le vieil homme, aveugle, avait délégué son fils unique, initié à son art.

9 Je pris alors l'habitude de rendre visite à ce vieux guérisseur (lia-kle-zan) réputé qui, n'ayant plus rien à prouver à ses pairs, se sentait libre de transmettre son enseignement à l'étrangère que j'étais. Parfois accueillie dans sa chambre, au milieu des objets puissants, je n'ignorais pas ses qualités de féticheurs ${ }^{5}$ (yu-kle-zan), mais ceci était affaire d'homme ; un jour, me promettait-il, je connaitrai la composition de certaines préparations puissantes ... un jour ... Une relation à plaisanterie, empreinte de tendresse, nous unissait.

10 Sans doute lui avait-on décrit mon matériel d'enregistrement et ses usages, il savait que je possédais une caméra. Il m'invita un jour à ne pas oublier celle-ci ${ }^{6}$ lors de la visite suivante, fixée un dimanche, car, me dit-il, son «apprentie» devait s'exercer publiquement à la danse dawa. Il souhaitait que je la filme. Nous découvrîmes alors tous que Zre était également zu-zan, devin-médium, puisque dawa est une des danses de divination gouro. Dans mon entourage Wagye se dansait un autre rituel qu'il m'avait toujours été strictement interdit de photographier.

\section{Histoire de Bwila et analyse du rituel dawa}

11 Réunir la somme nécessaire à l'achat du mouton sacrificiel ne fut pas facile car Bwila, «l'apprentie » (initiante) en question, réfugiée en cette tribu étrangère auprès de son thérapeute Zre, ne pouvait compter que sur la vente des produits du petit champ qu'elle cultivait seule; nous dûmes l'aider. Notre participation à la danse consista, en outre, à l'approvisionnement de l'assistance en vin de palme. Bwila fournit par ailleurs le riz et 
l'huile rouge $\mathrm{e}^{7}$ pour cuisiner la viande. Ce sont en effet les produits qu'exigent les $z \mathfrak{u}$, dieux tutélaires, avec lesquels Bwila, devra tenter d'entrer en communication dans la danse. "Souffles des ancêtres", intercesseurs auprès du principe créateur ${ }^{8}$, bali, pour l'attribution du souffle de vie donné à la naissance, les zu accompagnent le double invisible de tout être $(\mathbf{l e i})^{9}$; ils président à sa destinée. Selon leur nature, noirs (protecteurs) ou rouges (agités, dangereux), ils induisent des comportements posés et bénéfiques, la maîtrise d'arts ou de compétences, la fécondité, ou suscitent l'infortune par le désordre des conduites ${ }^{10}$. Mais on comprend que ces ancêtres retirent leur protection à celui qui néglige de les honorer et, pire, transgresse les lois qu'ils ont édictées. Toute maladie, tout malheur, valent comme rappel à l'ordre; ils invitent à déterminer la faute commise, par le recours aux oracles ou, plus directement, en faisant appel à ceux qui sont susceptibles d'entendre leurs voix: les zu-zan. Les ancêtres du consultant passent par l'intermédiaire des ancêtres du médium pour faire part de leurs desiderata à leurs descendants. Les zu-zan sont ainsi capables d'entrer en communication avec leurs propres ancêtres, ce que les humains ordinaires vivent parfois en rêve, ou dans des circonstances exceptionnelles et dramatiques. Se situer ainsi aux marges de deux mondes sans en perdre la vie ou la raison n'est donné qu'à ceux qui ont hérité de puissances protectrices par leurs pères ${ }^{11}$ ou à ceux qui les ont acquises au cours de longues errances, esquivant par la fuite les ordres inhumains de leurs zu. Les zu-zan qui résistent, sans en être brisés, aux épreuves traversées par ceux qui affrontent leur destin, reviennent chargés de la puissance des mondes où les zu les ont précipités, ceux de la brousse, des génies, des ancêtres. Ainsi Zre paya d'un mariage tardif et d'une maigre descendance l'exil de presque vingt ans que lui imposèrent ses $z u$, mais Gbo, le fils qu'il a initié, semble jusqu'à présent épargné.

Bwila, selon Zre, expie une faute commise autrefois par sa mère et sa sœur, toutes deux emportées avant d'avoir offert réparation. Le sort (les zu) s'en est pris à Bwila, ayant épuisé le reste de sa famille. Persécutée par les voix de ses zu, seule désormais, elle errait de guérisseurs en féticheurs lorsque la réputation de Zre parvint jusqu'à elle, qui appartenait à une autre unité territoriale, lointaine. Les habitants du village se souviennent encore des crises fréquentes qui la faisaient se rouler à terre, criant et délirant. Le diagnostic du guérisseur cependant était qu'il ne s'agissait pas là de folie ordinaire (gwèe), mais de la «folie de l'arc-en-ciel » (bomlin gwèe), lequel, comme chaque Gouro le sait, est l'émanation d'un puissant être de la brousse en forme de serpent python, logé dans certaines termitières. On reconstitua la faute originelle. La mère et la sœur de la malade, parties récolter les délicieux champignons de termitières ${ }^{12}$, avaient par hasard découvert des objets fétiches, tout en cuir ouvragé, au sommet d'un de ces monticules. Elles les ont ramassés comme le veut la tradition, car il se peut qu'un des ancêtres se manifeste ainsi (il intervient ensuite en rêve pour préciser le rôle de l'objet et les interdits afférents). Mais rien de ceci ne se produisit. Il s'agissait en fait des propres objets puissants de l'arc-en-ciel, que celui-ci avait déposés au soleil pour les faire sécher. Il convenait de les rapporter bien vite. Pour d'obscures raisons, les deux femmes n'en firent rien. Cette négligence leur fut fatale. Bwila n'avait pas d'autre issue, pour survivre, que d'acquérir elle-même une puissance capable de soutenir celle du serpent arc-en-ciel, d'apprivoiser cette puissance de la brousse, en devenant elle-même zu-zan. Le traitement fut long et il fallut tout le talent de Zre pour en venir à bout.

Dans un premier temps, les remèdes visaient à la soustraire au pouvoir de ses persécuteurs avant de la renforcer pour qu'elle puisse les côtoyer sans risques. Mais ceci 
ne fut pas suffisant car la maitrise requise devait lui permettre d'initier elle-même la communication $^{13}$. Il fallait ensuite entendre (car seules leurs voix sont perçues), et oser restituer aux consultants la teneur du message. Bwila n'avait pas encore réussi à mener de danse à ce terme.

Zre allait l'orienter dans cette nouvelle tentative, mais, vieux et aveugle, il avait sollicité l'aide d'autres zu-zan, outre son propre fils et son neveu ${ }^{14}$. Une fois l'orchestre de tam-tam accompagné de la cloche métallique et du chœur des chanteuses en place, le maître de cérémonie disposa au centre de l'espace une poterie sans fond emplie d'eau, mais qui ne fuyait pas ${ }^{15}$, signe de sa puissance. Sur cette poterie que son neveu entoura d'un cercle de cendre, il plaça le fétiche hérité de ses ancêtres après les avoir priés en déroulant la litanie de leur histoire. On lui apporta ensuite les statuettes de bois représentant ses zu, les objets puissants qu'il allait porter (ceinture, bonnet à grelots), ou tenir d'une main tandis que, de l'autre, il agiterait un bouquet de branchage de plantes « qui appellent les $z u »^{16}$. Il restait d'autres fétiches, laissés dans un van à disposition des danseurs. Bwila, tant qu'elle l'accompagnait au début de la danse, tenait à la main une petite pince de forgeron miniature, celle des ancêtres dont elle sollicitait la protection. Elle évolua désormais autour du cercle de cendre sans jamais y pénétrer, dans le demi arc de cercle que dessinaient orchestre et assistance, parfois rejointe par les autres zu-zan qui, eux, parcouraient plutôt l'espace laissé libre, scrutant l'horizon qui n'était vide que pour les yeux profanes («non retournés », non clairvoyants [yüe-zi-zan]).

15 Après quelques chants de louanges, Zre, d'une danse maîtrisée, retenue, accompagna les chanteuses, puis se rasseyant, délivra la parole oraculaire. Un jeune homme avait été ramené au village très malade, fils d'un autre féticheur et zu-zan, décédé récemment, dont il n'aurait pas perpétué la fonction. La maladie, interprétée comme manifestation du courroux du père, aurait dû être annoncée par l'apparition en rêve du vieil homme. La mère partie interroger son fils alité, on alla chercher l'oncle, lui-même guérisseur, aveugle et clairvoyant, pour conduire l'interrogation des médiums tandis que commençait le sacrifice, le vin de palme circulant à la ronde. À l'issue de cette première consommation Zre laissa la conduite du rituel à Bwila, qui désormais devait chercher, par sa danse malhabile, à entrer en contact avec ses $z u$. Bien qu'ayant troqué la pince de forgeron pour une queue-fétiche plus puissante confiée par l'oncle dès son arrivée, on la vit rechercher l'accolade avec Gbo et les assistants, qui lui crachotaient sur la tête ou sous la plante des pieds. Les objets qu'elle tenait, ainsi que les accolades et projection de salive mêlée de souffle d'hommes puissants, visaient à renforcer sa force vitale (nyale), attribut de son double invisible. Le souffle est en effet support de cette force vitale : souffle vital (kaa-beli-fuu ou bali-le-fuu) et force vitale (nyale) étant le double (lei) selon les exégètes (Haxaire 2003). Après des phases de piétinements denses devant l'orchestre, elle s'élança en pas rapides et amples vers l'espace extérieur où la suivirent les assistants. Le son de l'orchestre, singulièrement celui de la cloche métallique, avait attiré dans l'espace-temps ainsi délimité les $z u$, ancêtres invisibles, de chacun des membres de l'assistance. Ceux du malade s'adressèrent aux $z \mathfrak{u}$ de l'officiante qui revint porteuse d'oracles restitués d'une voix oppressée à la famille. Aucun des assistants ne participa à cette communication, bien qu'ils en eussent le pouvoir. Leur rôle ici semblait d'accompagner l'initiante qui s'aventurait dans le monde des zu en la protégeant de leur puissance ${ }^{17}$ : elle restait sans doute encore trop fragile pour affronter seule l'espace dégagé. Ainsi soutenue, Bwila put explorer l'ensemble des manquements reprochés; la famille, épuisée financièrement par les funérailles de cet homme chargé de fonctions et de cultes, avait en effet négligé 
certains sacrifices qu'elle considérait comme mineurs, dont celui des zu. Ceci transmis, Bwila pu s'entendre confier les reproches d'ancêtres d'autres personnes de l'assemblée, venues dans ce but, puis la cérémonie prit fin. Les tam-tams se turent, on rapporta les objets dans la chambre de Zre, Gbo souleva la poterie, laissant se répandre l'eau qu'elle contenait. Chacun reprit sa place, les ancêtres se retirèrent dans leur monde laissant aux vivants l'espace du village.

Maitrisant la communication avec ses $z u$, Bwila a pu repousser sa folie (gwée). Donnée comme «faisant tourner les yeux et la tête au point où, ne reconnaissant ni famille ni amis, alors en butte à une violence qui peut être meurtrière, le fou s'en va errer «en brousse ", l'aliénation, peut n'être qu'un signe de l'élection par les zu. Du moins est-ce l'espoir des familles de certains de ces malheureux vagabonds ; elles restent à l'affût des moindres prémonitions formulées par leur enfant, amorce d'une véritable fonction oraculaire.

\section{Principes organisateurs de la nosographie}

\section{Famille des « maladies-dans-la-tête »}

17 Les manifestations de $z \mathfrak{u}$ (ou gyina soit gbamgble-zu) entrent en effet dans la nosographie gouro comme " maladies-dans-la-tête » (wuo-gyi-dje) au même titre que gwée «la folie ", «la maladie du balai » (fanin) sorte de folie transmise par le balai posé comme fétiche protecteur, les crises de convulsion épileptiformes (gililai, yi-la-gbegbe, bali-le-dje) ou les convulsions dues à un empoisonnement (gùli-yu), les vertiges (yüe noonla), la détérioration des organes se situant au niveau de la tête, oreilles, yeux, nez (tone-wuli, yüe wuli, gnin-toro ), et, toutefois en spécifiant que cela fait tourner l'intérieur de la tête, les maladies résultant de la rencontre, ou de la non séparation, avec l'invisible et sa puissance (nyale et di).

Cet ensemble est bien distinct de celui des maladies qui font mal à la tête (wuo-yaa-dje) (migraines, rhume, pian, otites), de celles qui gonflent la tête (wuo-zii-dje) (caries, abcès...).

Toutes ces "maladies-dans-la-tête » altèrent, ou sont susceptibles, à terme, d'altérer ( gililai, convulsions épileptiformes) la capacité de se conduire "normalement » (tikli) en société.

Nous l'avons vu, dans $z u$, ces perturbations sont dues au mode de relation au monde induit par les ancêtres tutélaires de l'individu «malade ». Celui qui a un zu « rouge » ( gbamgble) demeure instable, bagarreur. Une femme sera incapable de s'attacher un mari, un homme de mener à bien une affaire.

21 Dans les maladies nyale et di, l'irruption du monde invisible et de sa puissance ne peut que fragiliser la stabilité de la personne. Les maladies nyale résultent de la rencontre avec le double invisible toujours à proximité du mort par accident que l'on découvre, tout comme de celui de l'animal puissant ${ }^{18}$ ou de l'ennemi que l'on vient de tuer. Leur double invisible suit le meurtrier ou la personne qui les a surpris, et cette dernière perd son chemin, tourne en rond, incapable de rentrer à la maison. Il faut posséder une poudre qui, mise dans les yeux du défunt, l'aveugle. A minima, lorsque l'on doit s'occuper d'un cadavre, respirer l'émanation de sa puissance risque de donner la toux et de tuer. On se détruit [la bouche et le nez] à respirer cette puissance (gnin-toro). Les troubles de di surviendraient si venait à manquer le rituel du même nom lors du deuil, moment où le 
veuf (ou la veuve) doit être lavé et recevoir dans les yeux un remède qui, de même, le masque aux yeux du double de son conjoint défunt, rendu alors incapable de le poursuivre en rêve. Sinon, «il voit la vie en noir, déambule sans raison en parlant tout seul, devient un peu fou ».

Une étymologie du nom de l'épilepsie gili-lai « conversation, causerie-poison » fait état de tentatives des ancêtres d'engager une causerie, toxique, avec leur descendant. L'étiologie des troubles équivalents chez les jeunes enfants (yi-la-gbegbe " eau-sur-tremblements ») serait d'avoir trop regardé l'eau, qu'eux-mêmes y jouent trop longtemps ou que, lorsqu'elle était enceinte, leur mère s'y soit mirée compulsivement. L'un des procédés de divination gouro utilisant les cuvettes d'eau, on peut se demander s'ils n'ont pas ainsi un peu trop titillé les ancêtres. Mais ici, le franchissement du miroir ne se fait pas, le malade, attiré par ses zu, résiste à les rejoindre ou à engager la communication.

Les yeux sont les reflets de l'intérieur, du double, nous ne le percevons pas chez les aveugles, pas plus que la parole extérieure n'atteint les sourds. Les organes sensoriels situés au niveau de la tête sont donnés comme porte d'entrée "dans la tête ». La projection particulière de liquide au niveau du visage (ban, tout comme arroser un sacrifice), à laquelle tout nouveau-né gouro est soumis dans les premiers mois de la vie, le prépare à ce mode d'administration de remède. Ainsi, le simple fait de respirer en présence de Klo, l'un des êtres de la brousse au remugle d'une violence à la mesure de sa puissance, tue. Son exhalaison est dite pénétrer dans la tête par le nez.

D'une façon ou d'une autre, ces « maladies-dans-la-tête » altèrent le discernement (gyi-kni ) dont l'organe est le cerveau pour les Gouro. Elles sont données comme conséquence ou cause d'un bouleversement (nonla) de l'interpénétration harmonieuse des registres du visible et de l'invisible dont toute déliaison signe la maladie puis la mort ${ }^{19}$. Voir l'invisible de son vivant, sans être soi-même ancêtre, laisse craindre qu'il ne s'agisse là d'un appel de l'au-delà et que ses jours sur terre ne soient comptés. Nous sommes certes doubles, comme insistent à le dire les vieux sages, mais, dans ce monde, seule la globalité de la personne peut être perçue.

Corps et double sont d'ailleurs pensés étroitement intriqués. À un premier niveau, le double « qui est le souffle qui est la force vitale nyale » s'articule au corps en circulant le long des mii (nerfs-vaisseaux-tendons), et s'ancre par le foie, organe distributeur du souffle de dieu. Mais plus encore, et de façon plus consubstantielle, certains organes comme le cœur, le foie, et le cerveau-moelle, faits de chair (le cœur), de sang coagulé (le foie), de graisse (le cerveau-moelle), qui répartissent et gardent en réserve les produits élaborés, constitutifs de l'humain, ont un rôle double ${ }^{20}$. Le cœur, répartissant le sang, est l'organe de la sensibilité, le foie, distribuant le souffle vital, celui des sentiments, des émotions. Enfin le cerveau, réserve de force, donne densité à la pensée. C'est l'organe de l'intelligence, du discernement. Comme si vie végétative et vie de relation se cumulaient en quelque sorte en chacun d'eux. Les qualités sensibles du monde physique, matériel, ont leur correspondance dans le monde des pensées, des émotions, des sentiments, des sensations. En d'autres termes, ces organes et les fluides qu'ils traitent (la graisse fondant) sont les supports matériels du double invisible et de ses composantes, qui les dédoublent effectivement. L'articulation double-corps nous apparaît comme une correspondance, un dédoublement en miroir où l'interdépendance des deux entités serait totale. racine à l'arbre, mais aussi densité à la pensée tout comme à la personne. 
27 Pour les Gouro, cerveau (zunu) et moelle des os, reliés par la moelle épinière, sont de même nature. Ils sont constitués d'une substance analogue à la graisse (gnonon) résultant de l'union des aliments et du souffle, qui vaut réserve de force. L'intérieur des os, donc la moelle, s'épuise lorsque l'on est fatigué ; le foie fond celle-ci pour impulser le souffle de la parole $^{21}$. On met en garde les enfants de ne pas dormir la tête vers le feu sinon leur cerveau fondrait. Mais le sommeil en lui-même induit, ou résulte, d'une fonte du cerveau qui devient alors perméable au sang qui se régénère et au souffle qu'il véhicule. Dieu renouvelle alors notre souffle vital à notre insu. Mais ce faisant, le sommeil libère le double, le désaliène en quelque sorte de la matérialité du corps mais aussi des contraintes du réel, et laisse place aux voyages du rêve. Cette perte de la raison (du discernement) peut se produire sous toutes autres conditions qui chaufferaient le cerveau, par exemple la colère ( «le foie bout »). Certains, lorsque la colère dépasse les bornes, se laissent aller à de bien étranges comportements. Les $z u$, dieux tutélaires, parcelles de souffle, sont donnés pour entourer (zii $z u$ ) ou suivre (zuo zu) la personne qu'ils protègent. S'ils s'avisent de pénétrer dans la personne, le double ne sait plus ce qui lui arrive, il part dans tous les sens (a yara-a gnan-gnan-gnan) ce que révèle le trouble des yeux (bli-bli «fous»). La puissance de ce souffle altère le cerveau. Une jeune femme ayant ainsi rencontré ses zu en était revenue la tête chamboulée (tournée : noonla), troublée, parlant à tort et à travers. Ces manifestations s'exaspèrent lorsque ce sont des êtres de la brousse qui vous saisissent, eux dont l'odeur forte traduit la puissance de l'émanation que l'individu respire et qui lui pénètre dans la tête.

On comprendrait que les thérapeutiques visent à empêcher l'intrusion d'un invisible menaçant en déployant une frontière masquant, cachant la personne ou en atténuant ses effets destructeurs en réduisant le différentiel des puissances. Il s'agit d'éviter la fonte, soit la dispersion, la déliaison de ce sur quoi s'appuie la personne pour distinguer (gyi kni) toute chose et construire son rapport au monde. Lorsque ce processus est déjà en œuvre, la cure devra tendre à rétablir cette base, ce fondement de densité (ces racines). En ce sens, mais il s'agit d'une interprétation de l'ethnologue ${ }^{22}$, le dispositif mis en place au départ du rituel, réaliser l'exploit de faire tenir un liquide dans une poterie sans fond, serait une métaphore du résultat espéré. L'eau du canari peut, bien entendu, rappeler celles des cuvettes utilisées pour la divination.

\section{Analyse des remèdes}

29 J'aimerais donc, tout comme F. Loux et F. Saillant (1995), convaincre les ethnologues de la pertinence, pour leurs propos, des études sur la phytothérapie et plus généralement la materia-medica eux qui, à l'instar d'Evans-Pritchard (1972), considèrent ce domaine trop technique et hors de leur champ. La logique qui préside aux soins, à l'organisation des recettes, apparait dans les allusions faites dans les commentaires accompagnant la transmission de recettes, toujours référées à la description du trouble qu'elles corrigent. Il est donc important de les recueillir dans leur intégralité et non dans leur seul déroulement opératoire. Mais ces remarques, succinctes, prennent sens parce qu'elles éclairent le déploiement des recettes associées, qui en retour valident la pertinence du commentaire. C'est donc un processus en boucle, entre pratiques et discours, qui, par touches successives, donne accès à la vision de la personne et du monde de la société à ce moment de son histoire. Nous analyserons ces données au regard de ce que nous avons compris du rituel et dans la vision de la personne et du monde gouro. 
30 Zre, pour guérir Bwila et lui permettre de deviner (monin do), nous dit ${ }^{23}$ lui avoir préparé des macérations laissées au soleil de bwi-la-monin-yiri «arbre de la lune, de savane » ( Gardenia sp.) puis bouillies, les décoctions devant être bues et le reste utilisé en lustrations pendant sept jours.

31 Il y a ajouté les feuilles de zaa-yiri «arbre de zaa, la foudre » (Lophira lanceolata) et de mlinnin (Parkia biglobosa) ainsi que les écorces de sept plantes quelconques, mais ayant poussé sur des termitières.

32 Bwila reçut en outre des instillations oculaires de kolebo-bé-gonin «le gros pouce du crabe » (indet.) pour lui donner la clairvoyance et le pouvoir de bilocation. C'était la première fois que j'entendais parler de cette plante que Gbo, le fils de Zre, ne connaissait pas lui-même.

33 Suivant la méthode déjà tentée précédemment (Haxaire 1994 et 1996) je vais dérouler le sens que prend la recette appliquée à Bwila par Zre, au regard des autres usages des plantes entrant dans sa composition.

34 - Les sept écorces de plantes poussées chacune sur une termitière sont de ce fait dotées de la puissance de ces monticules fétiches, ouvertures vers l'intérieur de la terre, domaine des $z u$ et des êtres de la brousse comme le serpent arc-en-ciel. L'écorce, contenant la sève, homologue du sang support de nyale, est une des parties de la plante qui concentre cette puissance. Sept est le chiffre de la complétude homme-femme [trois plus quatre ou quatre plus trois selon les régions gouro], c'est-à-dire de la toute puissance.

Cette composition est utilisée en décoction pour laver les enfants ayant bo-tin-le-gililai « le gililai de la diarrhée rouge », les convulsions dues à la diarrhée rouge, par une grande guérisseuse (554) « qui fait un peu comme la folie ». Le remède est dit « déplier » les « nerfs »- vaisseaux tendons que l'on sait collés et desséchés par la déshydratation au point de tirer en quelque sorte l'enfant comme une marionnette manipulée de l'intérieur. " Alors ses yeux restent grand ouverts mais calmes et il n'a pas l'écume aux lèvres ».

Les guérisseurs explicitent bien les effets recherchés de cette partie de recette : conférer au malade la puissance que ces plantes ont acquise en poussant sur des termitières. Elle permet la restauration du réseau le long duquel circulent les fluides vitaux dont le souffle vital, premier atteint dans toute maladie selon les vieux sages.

- Monin-yiri, «l'arbre de la lune » (Gardenia sp.) qui pousse en savane, se distingue de la variété de forêt (noire : sombre) par la couleur « blanche » de son écorce, de ses feuilles, et de ses fruits bien plus gros que ceux de la forêt. « La couleur de l'écorce ressemble un peu à celle de la lune ».

37 La recette de Zre utilise la décoction de tiges feuillées administrée tant en externe (en bain) qu'en interne (bue).

Les tiges feuillées de ce Gardenia de savane sont préparées en décoction, avec za-gon ( Asparagus sp.), dans la maladie djea ou djela « funérailles » (due au courroux des ancêtres lorsqu'un impair a été commis lors des funérailles, si, par exemple, un descendant s'allie avec un de leurs ennemis). On boit la première eau avant de faire un bain de vapeur et de se laver. Puis on mange la pâte salée de feuilles pilées (987). 
Un spécialiste des maladies de la petite enfance (969) ramasse les branches sèches de ce Gardenia comme remède de wuo " tête » (maladie des nouveau-nés qui creuse la fontanelle). Il l'ajoute d'une part au décocté utilisé en bain de vapeur puis en lustration. Il en prépare ensuite un charbon qu'il mêle à de l'huile rouge de palme pour en « attraper » la tête de l'enfant [pincer entre le pouce et l'index le haut du crâne comme pour refermer-ressouder les os de la fontanelle].

Une guérisseuse (224-225) prépare de même, avec de l'huile rouge, un charbon à partir des fruits secs de ce Gardenia contre les crises épileptiformes gililai. Dans ce cas, on en pince la fontanelle mais aussi le front, l'arrière de la nuque, la pointe du « cœur» (au niveau du sternum), le dos et les reins. Tandis que l'on ajoute les tiges feuillées dans la décoction de galabuanin « arbre à bois dur » pour faire un bain de vapeur sur la tête avant de laver le malade avec.

Toutes ces recettes comportent des bains agissant au niveau de l'enveloppe qu'est la peau pour les Gouro, mais ils s'accompagnent aussi d'administrations localisées de charbon sur les articulations (riches en nerfs-vaisseaux-tendons où circulent les fluides); tandis que les pâtes ou décoctés per os agissent en interne.

Il s'agit, pour deux entités pathologiques au moins (djela, gililai), de protéger le malade de l'atteinte des ancêtres tout comme dans $z u$ (par les remèdes externes), et parallèlement de renforcer les « organes contenants " que sont le crâne (dans wuo) et le réseau où circulent les fluides ainsi que le cœur qui les distribue (dans gililai).

Remarquons que les deux arbres de savane dont les tiges feuillées étaient utilisées dans le rituel pour « appeler les $z u$ » sont ici donnés comme remède, mais Zre en parle comme de vrai « fétiches » au détour de la recette.

41 - Zaa-yiri, «l'arbre de zaa », (Lophira lanceolata), porte ce nom parce qu'il est très souvent touché par la foudre (zaale) qui lui confère peut-être ses qualités.

\section{Tiges feuillées}

Zre (1088) utilise les tiges feuillées préparées avec le « dana des Gouro » (Acacia sp.) et une troisième plante non spécifiée, comme antidote des fétiches ou poisons lancés " yu duu ». C'est un yu bai, c'est-à-dire une protection. La recette comporte les feuilles pilées, séchées au soleil, réduites en poudre, tamisées et mises dans de l'huile. On s'oint le corps pour que le poison ne puisse pénétrer et coule sur la peau avec la transpiration. Ainsi ce poison ne tue jamais.

Elles sont également utilisées pour traiter Djekwasofuunin, l'ictère-anémie, pensé comme une consomption de l'intérieur par la puissance du sperme non évacué de l'utérus et diffusant sa puissance dans les vaisseaux d'une femme dont l'aménorrhée révèle la sécheresse donc la chaleur, et à qui, de ce fait, on avait interdit tous rapports sexuels (Haxaire 1999).

On boit la première eau d'un décocté préparé avec les tiges feuillées de « l'arbre de zaa », d'une plante non nommée, de ble (Annona senegalensis), de « l'arbre à bois dur noir » zin-ti (Bridelia ferruginea), de mlinin (Parkia biglobosa), avant d'en faire un bain de vapeur puis de s'en laver. 
Ces mêmes tiges feuillées sont utilisées dans les décoctions buanin (« pour faire grossir les enfants »), qui servent à masser puis laver et laisser baigner le nouveauné, mais ce terme générique désigne tout un ensemble d'indications (214).

\section{Écorce}

Contre zoo, « la sorcellerie », Zre utilise (1089) l'écorce mise dans un canari avec des boules de tiges feuillées de dana (Acacia sp.) pour faire un décocté utilisé en bain de vapeur, on se lave ensuite avec ce décocté pour faire évacuer.

C'est également cette partie de l'arbre que Zre emploie contre la maladie mi-nya « brancard utilisé pour le transport de cadavre ». On brûle l'écorce de zaa-yiri avec un morceau du bois du brancard et un morceau d'une natte sur laquelle un cadavre a été exposé, on en fait du charbon que l'on passe sur les articulations et au niveau du cœur (de la pointe du sternum). Le malade perd alors sa raideur, redevient souple (1100).

On le lave ensuite avec l'eau dans laquelle on a frotté des tiges feuillées de yi-to-bai (syn. bodo-bla) « les feuilles à faire les canaris » (Mondia whitei), toujours dans le même but (1101).

Et on le masse avec une pâte constituée des racines de wan yiri « la plante fourmi cadavre » (Securidaca longepedonculata) dans laquelle on a ajouté de jeunes feuilles de palmier (1102).

Un autre guérisseur (804) le donne comme remède de klo, le pian qui détruit le nez (et l'on " parle du nez »). Cette maladie peut être due à la rencontre avec le plus grand des génies de brousse mais également à la contamination par l'objet puissant protecteur préparé avec une coquille d'achatine (Achatina achatina : gros escargot). L'écorce pilée et séchée au soleil, humectée du suc de nunui-din (Physalis angulata) est réduite au feu dans un vieux canari jusqu'à ce que cela fasse un charbon que l'on passe sur le nez. On prépare ensuite un charbon avec les fleurs séchées au feu de vlonin (Spathodea campanulata) qu'on lui passe au cou, aux joues et au visage.

Zre (1118) possède quelques recettes vétérinaires dont celle de la " folie des chiens » (blin gali). L'écorce de zaa-yiri, les racines d'un dabla (Combretum racemosum) poussé sur une termitière (" ces racines sont de vrais fétiches ») sont posées dans un canari sur quatre rouleaux d'arbre à bois dur (galabuanin), on en fait une décoction sur laquelle on fait prendre au chien un bain de vapeur. Puis on prend les jeunes plants de la banane kola-man-kya (Musa sp.) qu'on lui fait goutter dans les yeux. « Cela enlève la folie de sa tête ».

\section{Bourgeons}

Dans kolu-kyekye, la toux « coqueluche ». Chauffés près du feu, on ajoute du sel et on se rend au milieu d'un carrefour pour le mettre dans la bouche de l'enfant. Il vomit alors ses glaires. (1067) 


\section{Feuilles}

Une guérisseuse (254) utilise les feuilles du néré pour prévenir les problèmes au moment de l'accouchement, lorsqu'ils sont dus à un adultère incestueux (dola : « quand tu as pour amie la femme d'un de tes parents »). On y ajoute les feuilles de bo-le-goi (Paullinia pinnata), « la cola du rat palmiste », et sept petits insectes « œil de la terre ", qui font des trous dans la terre. On fait sécher le tout et c'est ce qu'il faut se passer sur le pénis avant de lui faire l'amour.

Bali-le-dje « la maladie de dieu » distinguée de la « maladie de l'oiseau » (peut-être le tétanos dans certains cas) par la réussite du traitement peut être soignée par la recette suivante, transmise en rêve (943) : Les feuilles de mlinin (Parkia), et celles de dri-le-we (Uraria picta), dje-le-vlo-tan-yiri (indet.), de dabla (Combretum molle) et de tonan-tin (Amaranthus spinosus), sont froissées dans de l'eau à laquelle on ajoute un peu de vin rouge avant d'y adjoindre mone-bo, « un excréta de lune » brillant. On fait asseoir l'enfant sur un canari renversé et on lui verse le liquide sur la tête.

On pourrait compléter cette recette par la projection de quatre feuilles de citronnier mâchées avec quatre graines de maniguette.

Pour un autre guérisseur, (1058) les feuilles de Parkia sont utilisées en sus de la décoction d'autres plantes (1058) lorsqu'un adulte a été empoisonné soit par le poison spécifique kle soit par zuu-yu, « le fétiche du margouillat » et que « c'est grave, qu'il s'est arqué en arrière et que ses yeux sont fermés ». Si on lui projette dans le visage l'eau dans laquelle on a frotté ces feuilles, il sursaute puis s'assoit.

Pour Zre (1031), les feuilles du Parkia, plus les feuilles de kuli-dje-yiri, «l'arbre qui tue les tortues » (Cussonia barteri), et de boo (indet.), amollies sur un feu de paille, sont utilisées pour masser les enfants qui ont mal à la poitrine d'un seul côté (gole-ba-du).

\section{Écorce}

Quand on « tape » (empoisonne) quelqu'un avec gola-yu, le fétiche du chimpanzé. On prépare la décoction de l'écorce et on en fait des bains de vapeur puis une lustration pour que le malade s'arrête de crier.

La recette comprend, en outre, des feuilles de bwi-ziri (Vernonia guineense : amer comme ziri l'Erythrophleum, le poison d'épreuve) frottées dans de l'eau, cette eau étant projetée sur le malade, toujours pour qu'il s'arrête de crier.

Revue d'ethnoécologie, 1 | 2012 
ces plantes, pour partie « fétiches» donc puissantes (support de nyale) peuvent parfois être ingérées. Ce sont bien les sept écorces de plantes poussées sur sept termitières qui renforcent véritablement la puissance de la malade. dans-la-tête » dont la folie, notons que nous ne retrouvons pas, contre cette folie des $\mathbf{z u}$, ces plantes «noircissantes » telle kwin-la-moné (Eclipta prostrata) qui masquent la personne aux yeux de son conjoint ou de sa victime dans di ou nyale, et rencontrons peu d'usages des plantes odorantes (Citrus ou Securinega) qui font en quelque sorte un bouclier d'odeur. Zre emploie ces dernières contre les folies simples. C'est essentiellement de renforcement de la force vitale (nyanle) qu'il est question lorsque les zu sont en cause, tout comme nous 
avons pu le montrer dans l'analyse des transferts d'objets et de la scénographie des parcours d'assistants lors du rituel.

\section{Conclusion}

Les résultats de cette analyse semblent en cohérence avec ce qui avait pu être déduit de l'observation du rituel. Les données se répondent et sont susceptibles de s'informer réciproquement. Si je ne puis affirmer que la recette qui m'a été confiée est complète, c'est bien que ces remèdes ont, pour les acteurs, une valeur essentielle, au cœur du dispositif. Analyser la pharmacopée comme un système, selon la formulation de Dagognet (1964), ce que font F. Loux et F. Saillant, permet de dégager les " éléments organisateurs » qui fondent les recettes plus prosaïques et banales, et resituer le contexte dans lequel ces « grands remèdes » prennent sens.

Je n'ignore pas que pour que Bwila ait pu se restructurer un tant soit peu autour de sa nouvelle fonction, il a fallu qu'entre en jeu une dynamique intrapsychique et relationnelle, ce qui est évident lorsqu'on partage le quotidien de ses rapports à Zre qu'elle traite comme son père. Je n'ai pas choisi d'analyser ces dynamiques, pas plus que je n'ai pris pour objet le rituel thérapeutique comme lieu où se révèlent et se résolvent les conflits sociaux. Dans le rituel décrit, il n'était sans doute pas indifférent que le malade principal ait été le fils d'un autre zu-zan et féticheur décédé, ni que l'oncle intervenant se présente comme féticheur et clairvoyant, mettant en garde contre la présence d'une femme sorcière dans l'assemblée. La position dominante de Zre dans ce champ nous est apparue mais, ce village nous étant étranger, nous n'avons pu mesurer les enjeux des différentes interventions.

C'est bien l'organisation de la pharmacopée qu'il m'a fallu éclaircir dans un premier temps. Procéder ainsi, par exploration progressive de l'inconnu, résulte sans doute de ce que d'aucuns renvoient à l'équation personnelle du chercheur. Cette démarche assez peu partagée nécessite, il est vrai, de patientes collectes, car le sens se dégage de nombreuses occurrences et ces manipulations de matériel et de données peuvent, par leur caractère fastidieux, décourager beaucoup d'entre nous. Mais il y a du plaisir à voir se dégager du sens de matériaux épars, ce qui fut pour moi le cas lorsque je repris ma base de données pour analyser cet ensemble de traitements. Je n'avais pas, jusqu'alors, pris la mesure du fait qu'il s'agissait de maladies dans la tête, de là se reconstitua le puzzle. Se donner accès à ce savoir encyclopédique permet de suivre le cheminement de pensée de nos interlocuteurs. La démarche holistique, globale, qui caractérise l'anthropologie de la santé, ne peut, au risque précisément de rester partielle, négliger l'analyse de ces pratiques.

\section{BIBLIOGRAPHIE}

Boiteau P. 1972 - Qu'est-ce que l'ethnopharmacologie. La Pensée, 164 : 65-78. 
Dagognet F. 1964 - La Raison, et les remèdes. Paris, PUF, 347 p.

Deluz A. 1987 - Social and Symbolic Value of Feminine Knè Initiation among the Guro of Ivory Coast. In Parkin D. \& Nyamwaya D. (Ed.), Transformation of African Marriage. Manchester, Manchester University Press for the International African Institute :176-217.

Etkin N. 2001 - Perspectives in ethnopharmacology : forging a closer link between bioscience and traditional empirical knowledge. Journal of ethnopharmacology, 76 (2) : 177-182.

Evans-Pritchard E.E. [1931] 1972 - Sorcellerie, oracles et magie chez les Azandé, Trad. Evrard L. Paris, Gallimard, 642 p.

Grenand P., Moretti C., Jacquemin H. \& Prévost M.F. 2004 - Pharmacopées traditionnelles en Guyane. $2^{\text {ème }}$ éd., IRD Éditions, 816 p.

Haxaire C. 1994 - La femme adultère et le palmier : esquisse pour une anthropologie du remède. Écologie Humaine, 12 (1) : 3-28.

Haxaire C. 1996 - Thérapeutique préventive de l'amaigrissement des nouveau-nés chez les Gouro de Côte-d'Ivoire : variabilités et dérives. In Schröder E., Balansard G., Cabalion P., Fleurentin J. \& Mazars G. (Ed.), Médicaments et aliments, approche ethno-pharmacologique. Medecines and foods, the Ethnopharmacological Approach. IRD Éditions : 68-86. (Colloques et séminaires).

Haxaire C. 1999 - Remèdes de jouvence, remèdes de femme, essai d'analyse d'une pharmacopée dans une culture de tradition orale. In Guerchi A. (Ed.), Incontri tra Medicine, Meeting between Medicines. Genova, Erga : 199-216.

Haxaire C. 2003 - Âges de la vie et accomplissement individuel chez les Gouro (Nord) de Côted'Ivoire. L'Homme 167-168 : 105-128.

Haxaire C. 2005a - Lire, entendre, voir : modalités de la communication avec l'invisible et pouvoirs en pays gouro (RCI). Colloque international Prévoir et prédire la maladie. De la divination au pronostic : savoirs, pratiques, techniques, organisé par l'AMADES, 2-5 mars 2005, Ascona (Locarno), Suisse.

Haxaire C. 2005b - La danse de divination de Bwila. Colloque Prévoir et Prédire la maladie. De la divination au pronostic : savoirs, pratiques, techniques, Ascona, 3-5 mars 2005. (Vidéo montage SAVUBO (Université de Bretagne Occidentale).

Haxaire C. 2007 - Soins, toilette du nouveau-né et rites d'imposition du nom chez les Gouro de Côte-d'ivoire. In Bonnet D. \& Pourchez L. (Ed.), Du soin au rite dans l'enfance. IRD Éditions/Érès : 103-112.

Héritier F. 1996 - Masculin/Féminin. La pensée de la différence. Paris, Odile Jacob, 336 p.

Kerharo J. \& Bouquet A. 1950 - Plantes médicinales et toxiques de la Côte-d'Ivoire-Haute-Volta. Paris, Vigot Frères, 295 p.

Kerharo J. \& Adam J.G. 1974 - La pharmacopée Sénégalaise traditionnelle. Plantes médicinales et toxiques. Paris, Vigot Frères, 1011 p.

Lévi-Strauss C. 1962 - La pensée sauvage. Paris, Plon. 1990. Pocket, Paris, 347 p. (Agora).

Loux F. 1978 - Le jeune enfant et son corps dans la médecine traditionnelle. Paris, Flammarion. (Documents et essais).

Loux F. \& Richard P. 1988 - Le sang dans les recettes de médecine populaire. Mentalités 1 : 125-139. 
Loux F. \& Saillant F. 1990 - Pain et corps malade dans les recettes françaises et québécoises de médecine populaire. Uomo, 3 (1) : 179-195.

Loux F. \& Saillant F. 1995 - L'analyse comparative des recettes de médecine populaire en France et au Québec. L'exemple des rhumatismes. In Bouchard G., Segalen M. (Dir.), Dynamiques culturelles interrégionales au Québec et en France. Construction d'une enquête. Chicoutimi, Québec, Institut interuniversitaire de recherches sur les populations (IREP) : 157-180.

Mallart Guimera L. 2003 - La forêt de nos ancêtres. Vol 1 : Le système médical des Evuzok du Cameroun / Vol 2 : Le savoir botanique des Evuzok. Annales, Sciences humaines 167, Tervuren, Belgique, Musée Royal de l'Afrique Centrale, $511 \mathrm{p}$.

Portères R. 1961 - L'ethnobotanique : place, objet, méthode, philosophie. Journal d'agriculture tropicale et de botanique appliquée 8 (4-5) : 102-109.

Rilke R.M. 2002 - Lettres à un jeune poète. Grasset B. \& Biemel R. (Trad.). Grasset, 150 p. (Les Cahiers Rouges)

Saillant F. 1990 - Les recettes de médecine populaire. Pertinence anthropologique et clinique. Anthropologie et Société 14 (1) : 93-114.

Saillant F. \& Loux F. 1991 - Saigner comme un bœuf : le sang dans les recettes de médecine populaire québécoises et françaises. Une approche comparative. Culture 20 (1-2) : 151-163.

Turner V. 1972 - Les tambours d'affliction. Analyse des rituels chez les Ndembu de Zambie. Paris, Gallimard, 368 p. (Bibliothèque des Sciences humaines).

Wyman L.C. \& Harris S.K. 1941 - Navajo Indian Medical Ethnobotany. The University of New Mexico Bulletin. Anthropological series 3 (5) : 3-76.

\section{ANNEXES}


Tableau 1. Identification de la Materia medica

\begin{tabular}{|c|c|c|}
\hline Acacia pennata (Linn.) Willd. & & dana \\
\hline Acaciasp. & "dana des Gouro " & golo-dana \\
\hline Aframomun melegueta (Roscoe) K. Schum. & maniguette & süe \\
\hline Amaranthus spinosus L. & & tonan tin \\
\hline Annona senegalensis Pers. & & Ble \\
\hline Asparagus sp. sans doute Asparagus africanus Lam & "tige des discussions" ". & $z a-g o n$ \\
\hline Bridelia ferruginea Benth. & "le zin noir " & zin-ti \\
\hline Citrussp. & & golo lowuo \\
\hline Combretum molle R. Br. ex G. Don. & & dabla [gonin] \\
\hline Combretum racemosum $\mathrm{P}$. Beauv. & & dabla \\
\hline Cussonia barteri Seeman & "l'arbre qui tue les tortues» & kuli dje yiri \\
\hline Eclipta prostrata L. (syn : Eclipta alba (L.) Hassk) & "la lune sur les carnivores" & kwin la mone \\
\hline Elaeis guineensis Jacq. & palmier à huile & yo \\
\hline Erythrophleum guineense $\mathrm{G}$. Don & "poison d'ordalie " & ziri \\
\hline Gardenia sp. & "arbre de la lune, de savane ") & bui-la-monin-yiri \\
\hline Lophira lanceolata Van Tiegh. ex Keay & "arbre de zaa, la foudre " & zaa-yiri \\
\hline Mondia whitei (Hook. f.). Skeels & "les feuilles à faire les canaris " & $y i-t o-b a i=b o d o-b l a$ \\
\hline Musa sp. cult. & bananier $v t e ́$ & kola-man-kya \\
\hline Parkia biglobosa (Jacq.) R. Br. ex G. Don f. & néré & mlinnin \\
\hline Paullinia pinnata L. & "kola des rats palmistes" & bo-le-goi \\
\hline Physalis angulata $\mathrm{L}$. & & nunui-din \\
\hline Securinega longepedonculata Fres. & "arbre à odeur de fourmi cadavre " & wan-yiri \\
\hline Spathodea campanulata P. Beauv. & & vlonin \\
\hline Vernonia guineensis Benth. & "le ziri de savane" & bwi-ziri \\
\hline Uraria picta (Jacq.) DC. & "le sel des vaches" & dri-le-we \\
\hline indet. & & boo \\
\hline indet. & "le bois avec lequel dje danse vlo" & dje-le-vlo-tan-yiri \\
\hline indet, & "le gros pouce du crabe " & kolebo-bé-gonin \\
\hline générique & arbre à bois dur & galabuanin \\
\hline \multirow[t]{2}{*}{ Termitomyces } & champignon de termitière & \\
\hline & $\begin{array}{l}\text { sécrétion minérale soluble } \\
\text { "un excréta de lune " }\end{array}$ & mone-bo \\
\hline
\end{tabular}

Tableau 2. Identification des animaux cités

\begin{tabular}{|l|l|l|}
\hline & petits insectes « œil de la terre " & tre-yüe \\
\hline Achatina achatina syn. Achatina fulica Bowdich 1822 & achatine, gros escargot & klo \\
\hline Crotuta crotuta (Erxeleben, 1777) & hyène & glaun \\
\hline Kobus (Kobus) ellipsiprymnus (Ogilby, 1833) & cobe defassa & golo \\
\hline Loxodonta africana (Blumenbach, 1797) & éléphant & vui \\
\hline Panthera leo (Linné, 1756) & lion & djela \\
\hline Panthera pardus (Linné, 1756) & panthère & kua \\
\hline Syncerus caffer (Sparrman, 1779) & buffle & du \\
\hline Tragelaphus (tragelaphus) euryceros (Ogilby, 1857) & bongo & züin \\
\hline
\end{tabular}

\section{NOTES}

1. Pour Boiteau, embrasser ces deux dimensions nécessiterait « un corpus de connaissance d'un caractère beaucoup plus vaste [que la méthode chimiotaxonomique] et, tout d'abord, la connaissance de la langue. Il est souhaitable que le chercheur qui veut la mettre en œuvre ait une compréhension approfondie du groupe qu'il étudie et soit capable de s'y intégrer dans une certaine mesure...». Il s'agit de "pénétrer la nature profonde des mobiles des traditions populaires".

2. Et encore moins l'orientation vers les sciences humaines que Portères (1961) avait voulu donner à l'ethnobotanique, discipline dont il tentait de définir les bases, mais aussi posé par l'un 
des premiers ouvrages sur ce sujet, sinon le premier: Wyman, 1941 (nous devons ces renseignements au travail historique de D. Clément non encore publié)

3. Si nous nous en tenons aux chercheurs français à l'exception, par exemple, dans le domaine africain des travaux de L. Mallart (2003) et, chez les américanistes, de ceux des chercheurs en lien avec l'IRD de Cayenne (Grenand et al. 2004), chacun présentant néanmoins ses limitations car développant plus l'une de ces orientations, induite par les collaborations possibles (ou non) sur le terrain.

4. Pour F. Loux (1988) les éléments organisateurs sont « les éléments autour desquels le reste prend un sens" Il s'agit, dans le corpus français, d'étudier la façon dont se constituent les catégories de la pensée indigène, notamment le problème de l'appartenance d'un élément à plusieurs catégories.

Elle se propose de rester le plus près possible du «langage naturel ». Il a été retenu les items suivants : but, ou problèmes de santé traité par la recette, action thérapeutique ou préventive, subdivisée en plusieurs rubriques: type d'action sur le corps (appliquer, frotter, prier), ingrédients utilisés dans cette action (orties, fourmis, saint guérisseur...), circonstances et caractéristiques de l'action (caractère vivant des animaux utilisés ou couleur des ingrédients utilisés). C'est ce qui a été développé dans Saillant et Loux (1991).

5. Je reprends ici, par facilité de langage, la terminologie courante en français d'Afrique, que les ethnologues critiquent certes, mais ne savent remplacer autrement que par une glose qui serait, ici, suivant la traduction littérale des termes gouro, «celui qui fait des objets supports de puissance nyale» (cf. plus loin) et qui est lui même doté de cette puissance à un niveau remarquable.

6. Cet outil a permis d'analyser la scénographie en détail, tant du point de vue du déplacement des acteurs que du transfert des objets (Haxaire 2005a et 2005b).

7. Huile de palme, le palmier est l'arbre médiateur entre l'humain et son dieu créateur, on en parle comme du frère de l'homme.

8. Que nous traduirons désormais par dieu créateur, sachant qu'il est pensé comme réserve de souffle en quelque sorte.

9. Sur l'insistance de mes interlocuteurs, j'ai traduit par ombre-double cette partie invisible de l'homme, ce qui se justifie à l'analyse. Aux qualités dynamiques du corps correspond le souffle vital, tandis qu'à sa force, à sa chaleur, répond la puissance du nyale, force vitale.

Le souffle dont la puissance émane en nyale et qui contenu dans la personne prendrait la forme de son double invisible, ce souffle trouve son origine en dieu. Par le souffle, le corps s'articulait au double, par le souffle encore, le double lui-même se relie au monde du dieu créateur donc des ancêtres d'où il provient.

10. La notion gouro de la personne a été développée dans Haxaire (2003).

11. J'entends évidemment ce terme au sens de pères classificatoires.

12. Termitomyces.

13. Ces remèdes seront analysés plus loin.

14. zu zan eux-mêmes.

15. Pour ce tour de prestidigitation, dirions-nous, il disposait d'une poudre de feuilles mucilagineuses formant au contact de l'eau un film étanche.

16. Lophira lanceolata (zaa yiri) et de Parkia biglobosa (mlinnin)

17. Ce que l'analyse de la scénographie montre bien. De même, la vidéo nous permit-elle de travailler sur la circulation des objets entre les différents protagonistes.

18. Éléphant (vui), buffle (du), cobe defassa (golo), bongo (züin), hyène (glaun), panthère (kua), lion (djela).

19. La traduction littérale d'être malade ou « patraque » est « ne pas avoir son double sur soi ».

20. À la différence des organes "poche» comme l'estomac, les intestins ou les poumons qui traitent les nutriments bruts. 
21. Les testicules, de même, donnent la force au sexe.

22. Pour Zre (1034), les feuilles utilisées sont les remèdes d'une des maladies de sorcelleries, celle où les sorciers emplissent le ventre de liquide (ascites ?) [1034 est le numéro de référence de cette recette dans le corpus de $\mathrm{C}$. Haxaire. Vous pouvez consulter ce corpus sur demande auprès de l'auteur].

23. J'ignore si la recette confiée est exhaustive, mais ceci a peu d'importance pour la démonstration.

\section{RÉSUMÉS}

Les Gouro de l'unité territoriale Guo en Côte d'Ivoire pratiquent une danse de divination nommée Dawa que nous avons analysée en détail. Cette danse vaut rituel thérapeutique pour l'initiante, et sa réussite, la pleine restitution de la parole oraculaire, signe la maîtrise de la communication avec les ancêtres tutélaires (les $z u$ ) donc la détention d'une force suffisante pour résister à la folie spécifique, la « folie des zu », dont elle était atteinte. Le sens de ce rituel, ainsi que le déroulement de ce processus de guérison, peuvent certes se déduire de la vision du monde et de la personne Gouro. Mais les gloses données par les exégètes doivent être confrontées à l'analyse des pratiques : scénogrammes des acteurs participant au rituel, échanges d'objets puissants entre les acteurs, gestuelles et actes rituels. Les manipulations de plantes et l'ingestion de remèdes ne doivent pas être oubliées, bien qu'ils ne prennent sens que dans le savoir encyclopédique de la population qui pratique le rituel, savoir qu'il convient d'acquérir. Ici, l'analyse des recettes médicinales données pour traiter la «folie des $z u$ » chez les Gouro de Côte d'Ivoire, dans le contexte de l'ensemble des soins médicamenteux de la folie en général, a permis d'en dégager les «principes organisateurs » au sens donné par Loux et Saillant (1990). Elle joue alors le rôle de validation, confirmation ou critique, par les pratiques, des interprétations issues d'entretiens et d'observations d'épisodes de maladie et de rituels thérapeutiques présentés au préalable. L'étude des pharmacopées traditionnelles, entendues restituées dans l'organisation qui est la leur, vaut ainsi contexte empirique privilégié permettant de comprendre pleinement le sens des symboles rituels dont Turner (1972) demande de déployer les différentes facettes. Il est donc dommage qu'à l'instar d'Evans-Pritchard ([1931] 1972), nombre d'anthropologues travaillant sur l'infortune et la maladie aient toujours renoncé à suivre leurs interlocuteurs dans le détail des propriétés concrètes ou symboliques des plantes utilisées par les guérisseurs.

The Guro people in the Guo territorial unit in Ivory Coast practice a divination dance called Dawa, which we analysed in detail. This dance has the value of a therapeutic ritual for the novice: the success of the dance, i.e. full restitution of the word of the oracle, is a sign of mastery of communication with the tutelary ancestors ("zu") and thus of the possession of sufficient might to resist the specific madness - "zu madness" - that they would otherwise provoke. The meaning of this ritual and the progression of the healing process can indeed be deduced from the Guro vision of the world and from the Guro notion of a person. However, the glosses given by exegetes must be examined in the light of an analysis of practices, in other words, the scenogram: staging of the actors taking part in the ritual, exchange of powerful objects between the actors, ritual acts and gestures. The handling of plants and the ingestion of remedies must not be overlooked, even though these take on meaning only in the context of the encyclopedic knowledge of the population group that practices the ritual, knowledge that must be acquired. In the present case, 
the analysis of the medicinal formulas prescribed to treat "zu madness" among the Guro of the Ivory Coast, set in the overall context of medicinal treatment for madness in general, enables us to outline the "organisational principles" as defined by Loux and Saillant (1990). This analysis serves as a validation, confirmation or criticism - via the practices - of the interpretations drawn from interviews and observations of illness episodes and of therapeutic rituals presented beforehand. The study of traditional pharmacopoeias, restored in the full scope of their organisation, provides a privileged empirical context to fully understand the meaning of ritual symbols whose different facets need to be deployed, according to Turner (1968). It is unfortunate that, like Evans-Pritchard ([1931] 1972), many anthropologists who work on misfortune and illness, and all the more on rituals, did not seek further details of the material or symbolic properties of plants used by healers while following their interlocutors.

INDEX

Population Gouro/Guro

Mots-clés : folie, rituel de divination, rituel thérapeutique, anthropologie du remède, ethnopharmacologie, médecine - formules et recettes

Keywords : madness, divination ritual, therapeutic ritual, anthropology of medicinal treatment, ethnopharmacology, medicine - recipes and formulas

\section{AUTEUR}

\section{CLAUDIE HAXAIRE}

CESAMES (Centre de recherche Psychotropes, Santé mentale, Société)

CNRS (UMR 8136), Inserm (U 611), Université Paris Descartes

45 rue des Saints-Pères, 75270 Paris Cedex 06

Faculté de médecine de Brest (UBO), Département de Sciences Humaines et Sociales

CS 93837, 29238 BREST Cedex 3

haxaire@cassis-gw.univ-brest.fr 